\title{
Trapped surfaces and symmetries
}

\author{
Marc Mars ${ }^{1}$ and José M.M. Senovilla ${ }^{2}$ \\ 1 Área de Física Teórica, Facultad de Ciencias, Universidad de Salamanca, Plaza de la \\ Merced s/n, 37008 Salamanca, Spain \\ ${ }^{2}$ Departamento de Física Teórica, Universidad del País Vasco, Apartado 644, 48080 \\ Bilbao, Spain \\ E-mail: marc@usal.es and wtpmasej@lg.ehu.es
}

\begin{abstract}
We prove that strictly stationary spacetimes cannot contain closed trapped nor marginally trapped surfaces. The result is purely geometric and holds in arbitrary dimension. Other results concerning the interplay between (generalized) symmetries and trapped submanifolds are also presented.
\end{abstract}

PACS Numbers: 04.50.+h, 04.20.Cv, 04.20.Jb, 02.40.Ky

The importance of Penrose's concept of closed trapped surface, its usefulness, and the versatility of their applications is indubitable. To mention just a few outstanding situations where it has been essential, we can cite the development of the singularity theorems (see e.g. $[1,2,3,4]$ ), the general analysis of gravitational collapse and formation of trapped surfaces and black holes [5], the study of the cosmic censorship hypothesis [6] and the related Penrose inequality [7], or the numerical analysis of the Cauchy development of apparently innocuous initial data (e.g. [8] and references therein.)

In General Relativity, a trapped surface is a two-dimensional imbedded spatial surface such that the product of the traces of their two future-directed null second fundamental forms is everywhere positive. A more physical way of saying the same is that the two families of future-directed null geodesics orthogonal to the surface are, at least initially, simultaneously converging (or diverging). This concept can be easily translated to submanifolds of co-dimension 2 in any Lorentzian manifold $(\mathcal{V}, g)$ of arbitrary dimension $D$ (see e.g. $[9,10])$.

A spacetime will be called 'strictly stationary' if it contains a Killing vector field which is timelike everywhere. The term 'closed' is used for compact without boundary. In this letter we want to present a simple direct proof of the following result: closed trapped surfaces are absent in strictly stationary spacetimes. Obviously the same holds in those regions of a spacetime where there is a timelike Killing vector. A number of related 
results, some of them of more generality - concerning trapped submanifolds of any codimension or more general symmetries - , some others more specific - concerning static spacetimes-, and the formulae leading to them, are also presented. Our main result might seem intuitively obvious at first, as the area element of surfaces does not change along the timelike direction defined by the Killing vector in a strictly stationary spacetime. However, it is not straightforward: there are trapped surfaces in strictly stationary, and in static, spacetimes, even in the simplest examples. For instance, flat spacetime has trapped surfaces: for fully explicit examples see Example 4.1 in [4], p. 776. Thus, the requirement that the surface be closed is indispensable for our result.

To our purposes, and in order to keep the full generality, the best adapted definition of trapped surfaces is the one characterizing them through the use of their mean curvature vector $\vec{H},[9,10]$. The virtue of this characterization is that it can be easily generalized to imbedded submanifolds of any co-dimension. Thus, let $S$ be any $d$-dimensional $C^{2}$ imbedded spacelike submanifold in a causally orientable spacetime $(\mathcal{V}, g)$ (metric $g$ with signature $(-,+, \ldots,+))$ and let $\vec{H}$ be its mean curvature vector $[9,11,12] \ddagger$. We will assume, without further explicit mention, that all submanifolds $S$ are orientable. As usual we take $\overrightarrow{0}$ to be null (future and past) but not timelike or spacelike. In this letter we shall say that $S$ is

(i) future trapped if $\vec{H}$ is timelike and future-pointing all over $S$. Similarly for past trapped.

(ii) nearly future trapped if $\vec{H}$ is causal and future-pointing all over $S$ and timelike at least at a point of $S$, and correspondingly for nearly past trapped.

(iii) marginally future trapped if $\vec{H}$ is null and future-pointing all over $S$ and non-zero at least at a point of $S$, and analogously for marginally past trapped.

(iv) extremal or symmetric if $\vec{H}=\overrightarrow{0}$ all over $S$.

(v) absolutely non-trapped if $\vec{H}$ is spacelike all over $S$.

\section{Remarks}

- Definitions (i), (iv), (v) coincide with the standard ones in co-dimension two, while in that case (iii) is more general than the standard concept (e.g. [3, 4]) because both expansions may be non-identically zero on $S$ but with a vanishing product everywhere. Nevertheless, all the standard, non-extremal, marginally trapped $(D-2)$-surfaces are included in (iii).

- The above nomenclature is somewhat peculiar for the cases of co-dimension one or dimension one. In the first possibility $S$ is a spacelike hypersurface and, concentrating

$\ddagger$ The mean curvature vector can be defined according to several conventions. Our definition is such that $\vec{H}$ is the trace of the second fundamental form vector, without dividing by the dimension of the submanifold, and that it points outwards for a sphere in Euclidean space. 
on the future case for concreteness, (i) corresponds to a positive expansion everywhere on $S$, (ii) to a non-negative expansion which is positive at some point, (iv) to a maximal $S$, while (iii) and (v) are impossible. Similarly, $S$ represents a spacelike curve when $d=1$ and the concept of future trapping means here that the proper acceleration vector is past timelike along the curve for (i), null, past-pointing and not identically zero for (iii), and that $S$ is a geodesic for (iv).

The main tool in our reasoning is the known formula of the variation of the volume of any submanifold [11]. We present here a simple brief derivation adapted to our goals. Let $\Sigma$ be any smooth abstract $d$-dimensional manifold and let $\Phi: \Sigma \longrightarrow \mathcal{V}$ be a $C^{2}$ imbedding into the spacetime. We shall speak indistinctly of $\Sigma$ and its image in the spacetime $\Phi(\Sigma) \equiv S$ if no confusion arises. By means of the push-forward $\Phi^{\prime}$ one can define a set of $d$ linearly independent vector fields $\vec{e}_{A}$ on $S(A, B, \ldots=D-d, \ldots, D-1)$ which are tangent to $S$. Let $\gamma_{A B}=g\left(\vec{e}_{A}, \vec{e}_{B}\right)$ and $\boldsymbol{\eta}_{S}$ denote the first fundamental form and the corresponding canonical volume element $d$-form of $S$ in $(\mathcal{V}, g)$, respectively. Thus, the $d$-volume of $S$ is simply

$$
V_{S} \equiv \int_{S} \boldsymbol{\eta}_{S}
$$

We wish to know the variation of $\boldsymbol{\eta}_{S}$, and of $V_{S}$, when we perform a deformation of the submanifold $S$. To that end, let $\vec{\xi}$ be an arbitrary $C^{1}$ vector field on $\mathcal{V}$ defined on a neighbourhood of $S . \quad \vec{\xi}$ generates a local one-parameter group $\left\{\varphi_{\tau}\right\}_{\tau \in I}$ of local transformations, where $\tau$ is the canonical parameter and $I \subset \mathbb{R}$ is an interval of the real line containing $\tau=0$. We define a one-parameter family of surfaces $S_{\tau} \equiv \varphi_{\tau}(S)$ in $\mathcal{V}$, with corresponding imbeddings $\Phi_{\tau}: \Sigma \rightarrow \mathcal{V}$ given by $\Phi_{\tau}=\varphi_{\tau} \circ \Phi$. Observe that $S_{0}=S$. The corresponding first fundamental forms are simply $\gamma_{A B}(\tau)=\left(\varphi_{\tau}^{*} g\right)\left(\vec{e}_{A}, \vec{e}_{B}\right)$, with associated canonical volume element $d$-forms $\boldsymbol{\eta}_{S_{\tau}}$ (a $*$ denotes the pull-back.) Then, it is a matter of simple calculation to get

$$
\left.\frac{d \boldsymbol{\eta}_{S_{\tau}}}{d \tau}\right|_{\tau=0}=\frac{1}{2} \operatorname{tr}_{S}\left[\Phi^{*}\left(£_{\vec{\xi}} g\right)\right] \boldsymbol{\eta}_{S}=\frac{1}{2} \gamma^{A B}\left(£_{\vec{\xi}} g\right)\left(\vec{e}_{A}, \vec{e}_{B}\right) \boldsymbol{\eta}_{S}
$$

where $\operatorname{tr}_{S}$ denotes the trace in $S$ with respect to the induced metric and $£_{\vec{\xi}}$ is the Lie derivative with respect to $\vec{\xi}$. Another straightforward computation using the standard formulae relating the connections on $(\mathcal{V}, g)$ and on $(S, \gamma)$ (see, e.g. [12]) leads to

$$
\frac{1}{2} \operatorname{tr}_{S}\left[\Phi^{*}\left(£_{\vec{\xi}} g\right)\right]=\operatorname{div} \overrightarrow{\vec{\xi}}+\left.(\vec{\xi} \cdot \vec{H})\right|_{S}
$$

where $(\cdot)$ is the $g$-scalar product, div is the divergence operator on $S$ and $\vec{\xi}$ is the projection of $\vec{\xi}$ to $S$, that is to say, $\overline{\boldsymbol{\xi}}=\Phi^{*} \boldsymbol{\xi}$ so that $\bar{\xi}_{A}=\left.\left(\vec{\xi} \cdot \vec{e}_{A}\right)\right|_{S}$. These are the formulas we need, but for completeness we remark that from (1) and (2) one easily derives the expression for the variation of $d$-volume:

$$
\left.\frac{d V_{S_{\tau}}}{d \tau}\right|_{\tau=0}=\int_{S}(\operatorname{div} \overrightarrow{\bar{\xi}}+(\vec{\xi} \cdot \vec{H})) \boldsymbol{\eta}_{S}
$$


Lemma 1 If $\vec{\xi}$ is a Killing vector and $S$ is a closed imbedded submanifold, then

$$
\int_{S}(\vec{\xi} \cdot \vec{H}) \boldsymbol{\eta}_{S}=0
$$

Proof. If $£_{\vec{\xi}} g=0$, from (2) we obtain $(\vec{\xi} \cdot \vec{H})=-\operatorname{div} \overrightarrow{\vec{\xi}}$. Integrating this function over $S$, and using Gauss' theorem, the result follows as $S$ is compact without boundary.

Remark The geometric reason behing this result is that the $d$-volume of an imbedded closed submanifold is invariant under isometries of the spacetime. Note, however, that the proof above also holds if just the part of $£_{\vec{\xi}} g$ tangent to $S$ is traceless.

We arrive at our main result.

Theorem 1 If $(\mathcal{V}, g)$ is strictly stationary, then there are no marginally trapped, nearly trapped, nor trapped closed imbedded submanifolds in the spacetime.

Proof. We can assume that the timelike Killing vector, say $\vec{\xi}$, is future pointing. Then, if $\vec{H}$ pointed to the future (past) everywhere on $S,(\vec{\xi} \cdot \vec{H})$ would be non-positive (nonnegative) all over $S$, in contradiction with Lemma 1 unless $\vec{H}=\overrightarrow{0}$ everywhere on $S$.

\section{Remarks}

- Observe that this theorem implies, in particular, that there cannot be closed spacelike curves with a causal future acceleration vector in strictly stationary spacetimes, except for the extremal case of closed spacelike geodesics. Similarly, any closed spacelike hypersurface in a strictly stationary spacetime must have an expansion which changes sign, unless the hypersurface is maximal. The latter case is certainly possible. In fact, asymptotically flat maximal slices always exist in strictly stationary, asymptotically flat spacetimes [13] (see also [14] for generalizations to asymptotic stationarity).

- The extreme possibility $\vec{H}=\overrightarrow{0}$ can in fact happen in any dimension or co-dimension. Apart from what was said in the previous remark regarding closed geodesics or maximal hypersurfaces, there are also simple examples for the relevant case of codimension two. For instance, choose any $(D-1)$-dimensional proper Riemannian manifold containing a minimal surface $S$ and take its direct product with $\left(\mathbb{R},-d t^{2}\right)$. A more sophisticated yet explicit example for non-static spacetimes was presented by Newman in [15] for the famous Gödel spacetime (see also [9], pp.393-405).

By similar reasonings one can prove

Theorem 2 Let $S$ be closed and $\vec{\xi}$ any Killing vector which is null and nowhere vanishing on $S$. Then, $S$ cannot be trapped nor nearly trapped, and can be marginally trapped if and only if its mean curvature vector points along the same direction as $\vec{\xi}$ everywhere. 
Proof. Since $\vec{\xi}$ does not vanish on $S$ it follows that it is either future null or past null everywhere on $S$. For a (marginally, nearly) trapped $S,(\vec{\xi} \cdot \vec{H})$ is either non-negative or non-positive, therefore $\int_{S}(\vec{\xi} \cdot \vec{H}) \boldsymbol{\eta}_{S}=0$ implies $\left.(\vec{\xi} \cdot \vec{H})\right|_{S}=0$, so that $\vec{H} \propto \vec{\xi}$.

Remark A consequence of this theorem is that spacetimes representing pp-waves (which admit a covariantly constant and nowhere zero null Killing vector field $\vec{\xi}$, see e.g. [16]) do not admit closed spacelike hypersurfaces which are everywhere expanding (or contracting). In addition, there cannot be closed trapped or nearly trapped submanifolds and any closed marginally trapped submanifold $S$ must necessarily have a mean curvature vector parallel to $\vec{\xi}$. Therefore, $S$ must be contained in one of the null hypersurfaces orthogonal (and tangent) to $\vec{\xi}$.

Combining the previous theorems we get:

Corollary 2.1 Let $\vec{\xi}$ be any Killing vector on $(\mathcal{V}, g)$ and $S$ be any marginally trapped, nearly trapped, or trapped, closed submanifold. Then, (i) none of the connected components of $S$ can be fully contained in the region where $\vec{\xi}$ is timelike; (ii) $S$ can be within the subset where $\vec{\xi}$ is null and non-zero only in the case that $S$ is marginally trapped (and $\boldsymbol{\xi} \wedge \boldsymbol{H}=0$.)

Remark It can be easily proven that Theorems 1 and 2 and Corollary 2.1 also hold for immersed submanifolds. The details, however, are notationally cumbersome and we prefer to omit them.

The remark after lemma 1 allows for a relaxation of the Killing property for $\vec{\xi}$. As an illustrative example, among many others, we can use the so-called Kerr-Schild vector fields introduced in [17]. These are vector fields satisfying

$$
£_{\vec{\xi}} g \propto \boldsymbol{k} \otimes \boldsymbol{k}
$$

for a null vector field $\vec{k}$. Similarly, we could also use for $\vec{\xi}$ the more elaborated "causal symmetries" recently introduced in [18]. For the Kerr-Schild case we have $\frac{1}{2} \gamma^{A B}\left(£_{\vec{\xi}} g\right)\left(\vec{e}_{A}, \vec{e}_{B}\right) \propto \gamma^{A B}\left(\vec{k} \cdot \vec{e}_{A}\right)\left(\vec{k} \cdot \vec{e}_{B}\right)$ which has the sign of the proportionality function, or vanishes if $\vec{k}$ is orthogonal to $S$. In the former case, and if the proportionality factor has a sign on $S$, one can derive properties about the future or past trapping of the sets in a manner analogous to the one we shall use later for conformal motions. In the latter case, theorems 1 and 2 hold just the same for these Kerr-Schild vector fields. An explicit example of this situation is provided by the Vaidya radiating spacetime which has a proper Kerr-Schild motion $\vec{\xi}$ (see [17] p.667). This $\vec{\xi}$ is partly timelike, partly spacelike, and null at the frontier, which is the apparent horizon. One can easily check in this simple spacetime that the spherically symmetric closed trapped surfaces lie entirely in the spacelike region of $\vec{\xi}$, and that the apparent horizon is formed by marginally trapped spheres (see e.g. Example 4.2 in [4].) Actually, the Kruskal extension of Schwarzschild 
spacetime is contained here as the subcase with constant mass function, and then the vector field $\vec{\xi}$ coincides with the asymptotically stationary Killing vector.

Even if the part of $£_{\vec{\xi}} g$ tangent to $S$ is not trace-free one can obtain information from formula (2). Take the relevant example of conformal Killing vectors

$$
£_{\vec{\xi}} g=2 \phi g
$$

so that $\frac{1}{2} \gamma^{A B}\left(£_{\vec{\xi}} g\right)\left(\vec{e}_{A}, \vec{e}_{B}\right)=\phi d$. When $S$ is closed, integration of (2) provides

$$
\int_{S} \phi \boldsymbol{\eta}_{S}=\frac{1}{d} \int_{S}(\vec{\xi} \cdot \vec{H}) \boldsymbol{\eta}_{S}
$$

If $\vec{\xi}$ is timelike and $\left.\phi\right|_{S}$ has a sign - this includes, in particular, all timelike homothetic vectors - , then any (marginally, nearly) trapped closed $S$ must have a fixed causal orientation for the trapping. If $\left.\phi\right|_{S} \geq 0$, say, then $\vec{H}$ must be oppositely oriented to $\vec{\xi}$. Furthermore, extremal submanifolds are forbidden. As an illustrative example, take the obvious future timelike conformal Killing vector field of Robertson-Walker cosmological spacetimes. Application of the above reasoning immediately leads to the conclusion that, for expanding Robertson-Walker cosmologies, all possible closed trapped surfaces must be past-trapped, and future trapped if the spacetime is contracting, and in neither case there can be minimal closed surfaces, nor maximal closed hypersurfaces. Of course, this corroborates the usual elementary knowledge (see e.g. [4] p.779.)

Let us finally make some considerations about the particular case of strictly static spacetimes. In this case, there is a timelike Killing vector $\vec{\xi}$ which is integrable, that is $\boldsymbol{\xi} \wedge d \boldsymbol{\xi}=0$ so that $\vec{\xi}$ is orthogonal to a family of spacelike hypersurfaces. Being $\vec{\xi}$ a Killing vector without rotation, these hypersurfaces are all time symmetric in the sense that their second fundamental forms in $(\mathcal{V}, g)$ vanish. A partial generalization of this result to sets of arbitrary co-dimension is the following.

Proposition 1 Let $\vec{\xi}$ be a hypersurface-orthogonal timelike Killing vector. Then, all submanifolds contained in any one of its orthogonal hypersurfaces have a mean curvature vector which is everywhere spacelike or zero.

Proof. Let $S$ be the submanifold. As $\vec{\xi}$ is orthogonal to $S$, the tangent projection $\overrightarrow{\vec{\xi}}$ appearing in (2) vanishes. As the lefthand side of that equation also vanishes we get $\left.(\vec{\xi} \cdot \vec{H})\right|_{S}=0$, so that $\vec{H}$ must be spacelike or zero everywhere.

This result also follows directly by using the time-inversion isometries of a static spacetime. However, the proof we have presented (and therefore the proposition) holds for arbitrary submanifolds, closed or not, as long as they are orthogonal to $\vec{\xi}$, even if $\vec{\xi}$ is not hypersurface-orthogonal. It follows that all such submanifolds are absolutely non-trapped or extremal (or a combination). As a consequence, in strictly stationary 
spacetimes all possible (marginally, nearly) trapped surfaces-necessarily non-closed due to theorem 1 - must be non-orthogonal to $\vec{\xi}$. This case can certainly happen-e.g. the example of flat spacetime mentioned above-.

Proposition 1 and the example just before Theorem 2 show that closed extremal submanifolds (i.e. with vanishing mean curvature vector) can exist in static spacetimes when they are contained in a hypersurface orthogonal to the static Killing $\vec{\xi}$. A natural question is whether other types of closed extremal submanifolds can exist. Our last proposition shows that this is not the case, i.e. that all closed extremal submanifolds in a strictly static spacetime must lie within a hypersurface of constant static time.

Proposition 2 Let $\vec{\xi}$ be a strictly static Killing vector on $(\mathcal{V}, g)$ and $S$ a closed spacelike submanifold with vanishing mean curvature vector $\vec{H}=0$. Then, $S$ is contained in a hypersurface orthogonal to $\vec{\xi}$.

Proof. Let $t$ be the static time function of $(\mathcal{V}, g)$, i.e. $\boldsymbol{\xi}=-V^{2} d t$, where $V^{2}=-(\vec{\xi} \cdot \vec{\xi})$. Let also $\left.\tilde{t} \equiv t\right|_{S},\left.\tilde{V} \equiv V\right|_{S}$. Since $\vec{\xi}$ is a Killing vector it follows, as in the proof of Lemma 1 , that $(\vec{\xi} \cdot \vec{H})=-\operatorname{div} \overrightarrow{\vec{\xi}}$. Since $\bar{\xi}^{A}=-\tilde{V}^{2} D^{A} \tilde{t}$, where $D^{A}$ is the covariant derivative of $\gamma_{A B}$, we immediately derive that

$$
(\vec{H} \cdot \vec{\xi})=\gamma^{A B} D_{A}\left(\tilde{V}^{2} D_{B} \tilde{t}\right)
$$

If $(\vec{H} \cdot \vec{\xi})=0$ (in particular if $S$ is extremal) we have $\gamma^{A B} D_{A}\left(\tilde{V}^{2} D_{B} \tilde{t}\right)=0$, which can be viewed as an elliptic PDE for $\tilde{t}$ on a closed submanifold $S$. Uniqueness of solutions for this PDE except for an additive constant follows from standard results (see e.g. [19]). In our case this can be proven directly by integrating $D_{A}\left(\tilde{V}^{2} \tilde{t} D^{A} \tilde{t}\right)$ on $S$ and using Gauss' theorem. Consequently it follows that $\tilde{t}=c$, constant on $S$, which proves that $S$ must be contained in a constant static time slice of the spacetime.

Many other results may be obtained from formulas (1) and (2), which can be helpful in other studies too. An obvious open question from all the above, that we would like to remark, is: in strictly static spacetimes, are there any closed submanifolds with a causal $\vec{H}$ everywhere? This question is non-trivial even in Minkowski spacetime. A positive answer would imply, among other things, that there are closed spacelike surfaces with positive Hawking mass in flat spacetime. This could have interesting implications for the use of inverse mean curvature flows in spacetime as a tool for proving the Penrose inequality, specially if there are closed surfaces with $\vec{H}$ null everywhere. It would be interesting to settle this issue. It might be also worth exploring the possible applications of the concept of trapped submanifolds of any codimension to the problems in which the codimension two case has been important, such as those enumerated at the begining of this letter. 


\section{Acknowledgements}

We thank the anonymous referee for useful comments. JMMS acknowledges financial support under grants BFM2000-0018 of the Spanish CICyT and no. 9/UPV 00172.31014456/2002 of the University of the Basque Country. MM wishes to thank the Junta de Castilla y León for financial support under grant SA002/03

\section{References}

[1] R. Penrose, Gravitational collapse and space-time singularities, Phys. Rev. Lett. 14, 57 (1965).

[2] S.W. Hawking, R. Penrose, The singularities of gravitational collapse and cosmology, Proc. Roy. Soc. London A314 529 (1970).

[3] S.W. Hawking, G.F.R. Ellis, The large scale structure of space-time, (Cambridge Univ. Press, Cambridge, 1973).

[4] J.M.M. Senovilla, Singularity theorems and their consequences, Gen. Rel. Grav. 30, 701 (1998).

[5] P.N. Demni, A.I. Janis, The characteristic development of trapped surfaces, J. Math. Phys. 14, 793 (1973); U. Alfes, H. Müller zum Hagen, Spherically Symmetric Event Horizons and Trapped Surfaces Developing from Innocuous Data, Class. Quantum Grav. 11, 2705 (1994); R. Beig, N. Ó Murchadha, Trapped Surfaces in Vacuum Spacetimes, Class. Quantum Grav. 11, 419 (1994); R. Beig, N. Ó Murchadha, Vacuum Spacetimes with Future Trapped Surfaces, Class. Quantum Grav. 13, 739 (1996); P. Bizon, E. Malec, N. Ó Murchadha, Trapped surfaces due to concentration of matter in spherically symmetric geometries, Class. Quantum Grav. 7, 961 (1989).

[6] R. Penrose, Gravitational collapse: the role of general relativity, Nuovo Cimento 1, 252 (1969); R. Wald, Gravitational collapse and cosmic censorship, gr-qc/9710068; D. Christodoulou, The instability of naked singularities in the gravitational collapse of a scalar field, Ann. Math. 149, 183 (1999); A. Krolak, Towards the proof of the cosmic censorship hypothesis, Class. Quantum Grav. 3, 267 (1986); P.T. Chruściel, J. Isenberg, V. Moncrief, Strong cosmic censorship in polarized Gowdy spacetimes, Class. Quantum Grav. 7, 1671 (1990); M. Dafermos, The interior of charged black holes and the problem of uniqueness in general relativity, gr-qc/0307013.

[7] R. Penrose, Naked singularities, Ann. N. Y. Acad. Sci. 224, 125 (1973); G. Gibbons, Collapsing shells and the Isoperimetric inequality, Class. Quantum Grav. 14, 2905 (1997); G. Huisken, T. Ilmanen, The inverse mean curvature flow and the Riemannian Penrose inequality, J. Diff. Geom. 59, 353 (2001); H. Bray, Proof of the Riemannian Penrose inequality using the Positive Mass Theorem, $J$. Diff. Geom. 59, 177 (2001); R. Geroch, Energy Extraction, Ann. N. Y. Acad. Sci. 224, 108 (1973); P.S. Jang, R. Wald, The positive energy conjecture and the cosmic censorship, J. Math. Phys. 18, 41 (1977); M. Herzlich, A Penrose-like inequality for the mass of Riemannian asymptotically flat manifolds, Commun. Math. Phys. 188, 121 (1997); E. Malec, M. Mars, W. Simon, On the Penrose inequality for general horizons, Phys. Rev. Lett. 88, 121102 (2002); M. Ludvigsen, J.A.G. Vickers, An inequality relating total mass and the area of a trapped surface in general relativity, J. Phys. A: Math. Gen. 16, 3349 (1983); G. Bergqvist, On the Penrose inequality and the role of auxiliary spinor fields, Class. Quantum Grav. 14, 2577 (1997); E. Malec, N. Ó Murchadha, Trapped surfaces and the Penrose inequality in spherically symmetric geometries, Phys. Rev. D 49, 6931 (1994); S.A. Hayward, Quasi-localization of Bondi-Sachs energy-loss Class. Quantum Grav. 11, 3037 (1994).

[8] L. Lehner, Numerical Relativity: a review, Class. Quantum Grav. 18, R25 (2001); B.K. Berger, 
Numerical approaches to spacetime singularities, Living Rev. Relativity, lrr-2002-1 (2002); G.B. Cook, Initial data for numerical relativity, Living Rev. Relativity, lrr-2000-5 (2000).

[9] M. Kriele, Spacetime, (Springer, Berlin, 1999).

[10] J.M.M. Senovilla, Trapped surfaces, horizons and exact solutions in higher dimensions, Class. Quantum Grav. 19, L113 (2002).

[11] J. Jost, Riemannian Geometry and geometric analysis, 3rd ed. (Springer, Berlin, 2001).

[12] B. O'Neill, Semi-Riemannian Geometry: With Applications to Relativity (Academic Press, 1983).

[13] R. Bartnik, P.T. Chruściel, N. Ó Murchadha, On maximal surfaces in asymptotically flat space-times, Commun. Math. Phys. 130, 95 (1990).

[14] P.T.Chruściel, R.Wald, Maximal hypersurfaces in asymptotically stationary space-times, Commun. Math. Phys. 163, 561 (1994).

[15] R.P.A.C. Newman, Black holes without singularities, Gen. Rel. Grav. 21, 981 (1989).

[16] H. Stephani, D. Kramer, M.A.H. MacCallum, C. Hoenselaers, E. Herlt, Exact Solutions to Einstein's Field Equations Second Edition (Cambridge University Press, Cambridge, 2003).

[17] B. Coll B, S.R. Hildebrandt, J.M.M. Senovilla, Kerr-Schild symmetries, Gen. Rel. Grav. 33, 649 (2001).

[18] A. García-Parrado, J.M.M. Senovilla, Causal symmetries, Class. Quantum Grav. 20, L139 (2003); General study and basic properties of causal symmetries, gr-qc/0308091.

[19] D. Gilbarg, N.S. Trudinger, Elliptic Partial Differential Equations of Second Order (Springer-Verlag, 1983). 